Open Access

\title{
Tinkering signaling pathways by gain and loss of protein isoforms: the case of the EDA pathway regulator EDARADD
}

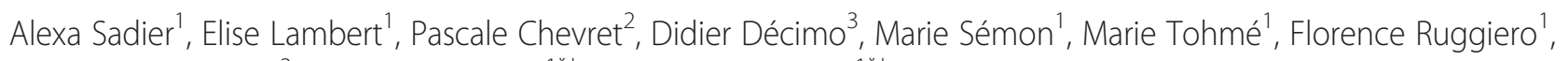
Théophile OhImann ${ }^{3}$, Sophie Pantalacci ${ }^{1^{*} \dagger}$ and Vincent Laudet ${ }^{*^{*}+}$

\begin{abstract}
Background: Only a handful of signaling pathways are major actors of development and responsible for both the conservation and the diversification of animal morphologies. To explain this twofold nature, gene duplication and enhancer evolution were predominantly put forth as tinkering mechanisms whereas the evolution of alternative isoforms has been, so far, overlooked. We investigate here the role of gain and loss of isoforms using Edaradd, a gene of the Ecodysplasin pathway, implicated in morphological evolution. A previous study had suggested a scenario of isoform gain and loss with an alternative isoform (A) newly gained in mammals but secondarily lost in mouse lineage.

Results: For a comprehensive view of A and B Edaradd isoforms history during mammal evolution, we obtained sequences for both isoforms in representative mammals and performed in vitro translations to support functional predictions. We showed that the ancestral B isoform is well conserved, whereas the mammal-specific A isoform was lost at least 7 times independently in terminal lineages throughout mammal phylogeny. Then, to gain insights into the functional relevance of this evolutionary pattern, we compared the biological function of these isoforms: i) In cellulo promoter assays showed that they are transcribed from two alternative promoters, only B exhibiting feedback regulation. ii) RT-PCR in various tissues and ENCODE data suggested that $B$ isoform is systematically expressed whereas $A$ isoform showed a more tissue-specific expression. iii) Both isoforms activated the NF-KB pathway in an in cellulo reporter assay, albeit at different levels and with different dynamics since A isoform exhibited feedback regulation at the protein level. Finally, only B isoform could rescue a zebrafish edaradd knockdown.
\end{abstract}

Conclusions: These results suggest that the newly evolved $A$ isoform enables modulating EDA signaling in specific conditions and with different dynamics. We speculate that during mammal diversification, A isoform regulation may have evolved rapidly, accompanying and possibly supporting the diversity of ectodermal appendages, while $B$ isoform may have ensured essential roles. This study makes the case to pay greater attention to mosaic loss of evolutionarily speaking "young" isoforms as an important mechanism underlying phenotypic diversity and not simply as a manifestation of neutral evolution.

\footnotetext{
*Correspondence: sophie.pantalacci@ens-lyon.fr; vincent.laudet@ens-lyon.fr

${ }^{\dagger}$ Equal contributors

${ }^{1}$ Institut de Génomique Fonctionnelle de Lyon, UMR 5242 du CNRS,

Université de Lyon, Université Claude Bernard Lyon 1, Ecole Normale

Supérieure de Lyon, 46 Allée d'Italie, 69364 Lyon Cedex 07, France

Full list of author information is available at the end of the article
} 


\section{Background}

Explaining how species share a common genetic toolkit, but at the same time show a high phenotypic diversity is a crucial question of evo-devo [1-3]. Many examples described and theorized in the last 30 years have identified the genetic basis for evolutionary changes such as the modification of the existing toolkit by mutation (in coding or regulatory sequence) or its expansion/constriction by gene duplication and loss [1]. However, with the rise of omics, other mechanisms that contribute to diversification of developmental gene products can now be analyzed in depth. Among them, the generation of alternative isoforms is particularly relevant $[4,5]$.

Isoforms are generated by alternative splicing sensu lato (i.e. via differential exon retention, alternative promoters and alternative $3^{\prime}$ splice site) which, is considered as the most prominent mechanism for generating mRNA structural complexity $[6,7]$. It can produce numerous new gene products that can be differentially regulated spatio-temporally and translated into proteins with different functions [8]. The evolution of alternative isoforms can give rise to new function or sub-function in different species [9]. Thus, the generation of alternative isoforms is now considered as an important force in evolution. Different levels of alternative splicing sensu lato were described among eukaryotes (and more precisely in the animal kingdom) [10-12]. Studies that compared transcriptomes between species or tissues have shown that a significant portion of splicing events are species-specific [5], suggesting a high turn-over in isoform gain and isoform loss and raising the question of whether the evolution of isoforms is largely neutral. However the extent to which this quantification is biased is unclear as datasets often incorporate unwanted splicing events like noisy splicing or regulatory splicing (e.g. the splicing of an alternative transcript does not result in encoding another protein but rather in regulating the abundance of another transcript [9]). Moreover, omics studies can only rely on very indirect and sometimes circular arguments (e.g. what is conserved is functional) to assess the functional significance of detected isoforms, if any and, the phylogenetic sampling is often very limited (e.g. mouse/human or a few mammals). In this context, such omics studies encounter difficulties concluding about the significance of patterns of isoform gain or loss during evolution. The field would now greatly benefit from specific case studies that combine functional experiments and broad phylogenetic sampling. Ideal candidates for such studies would be genes known to be important in phenotypic evolution, and that are likely to display variation in splice variants in a given group of species.

Edaradd is one such promising candidate [9]. It encodes the intracellular scaffold protein EDARADD (or adaptor) specifically involved in the EDA-A1 pathway. In all vertebrate species tested so far, this pathway is necessary to the normal formation of ectodermal appendages (i.e. teeth, scales, hair, feathers, glands...) that are either missing or malformed in loss of function mutants $[13,14]$. Since ectodermal appendages are hot spots of adaptation, this pathway is particularly relevant for morphological evolution [13]. It has been repeatedly found to be involved in morphological evolution, in species as distant as the stickleback fish and humans $[15,16]$. Briefly, this pathway is related to the TNF receptor signaling pathway (Fig. 1A) and is composed by three specific components: the ligand EDA-A1, the receptor EDAR and the adaptor EDARADD. EDARADD serves as a relay between the receptor activated by the ligand and other intracellular proteins (such as TRAFs) involved in NF- $\mathrm{kB}$ pathway regulation, resulting in the activation of the NF-kB-dependent transcription upon ligand binding [17]. In a previous study we have shown that all three corresponding genes of the EDA-A1 pathway are highly conserved in vertebrates [18]. Most interestingly, in the context of this study, we had noted that, while non-mammalian genomes only carry a single isoform for the EDARADD adaptor, in mammals this gene encodes two isoforms, called A and B. The two isoforms differ by their short $\mathrm{N}$-terminal part encoded by alternative first exons for detail on the genomic region see Additional file 1: Figure S1. The B isoform more closely resembles the isoform found in chicken, although the limited sampling associated with the short size of the B peptide did not allow drawing firm conclusions [18]. We also noted that B isoform is the only isoform found in mouse and rat [18]. These data therefore suggest a complex history of gain and loss with a newly evolved isoform in the mammalian lineage (A isoform), which was secondarily lost in the mouse/rat lineage.

Thus the Edaradd gene would provide a case study for the gain and loss of isoforms expressed from alternative promoters in relation to phenotypic evolution. It is quite obvious that isoform gain and evolution could support phenotypic evolution (and notably phenotypic innovation), provided that isoforms have different function or regulation. Because the functional tests to establish the function of EDARADD (mentioned above) were carried out in mouse, where the isoform A is absent, or in human, selecting the human-mouse conserved B isoform, it is currently unknown whether the two isoforms have a similar or a very different function. It is hard to predict just from their sequence. On one hand, in terms of domain content, both isoforms are very similar (Death domain, TRAF binding domains), but on the other hand, a very short N-terminal peptide is in principle sufficient to confer different biochemical properties, such as different levels of activation or different downstream signaling. Moreover, in silico predictions [18] 


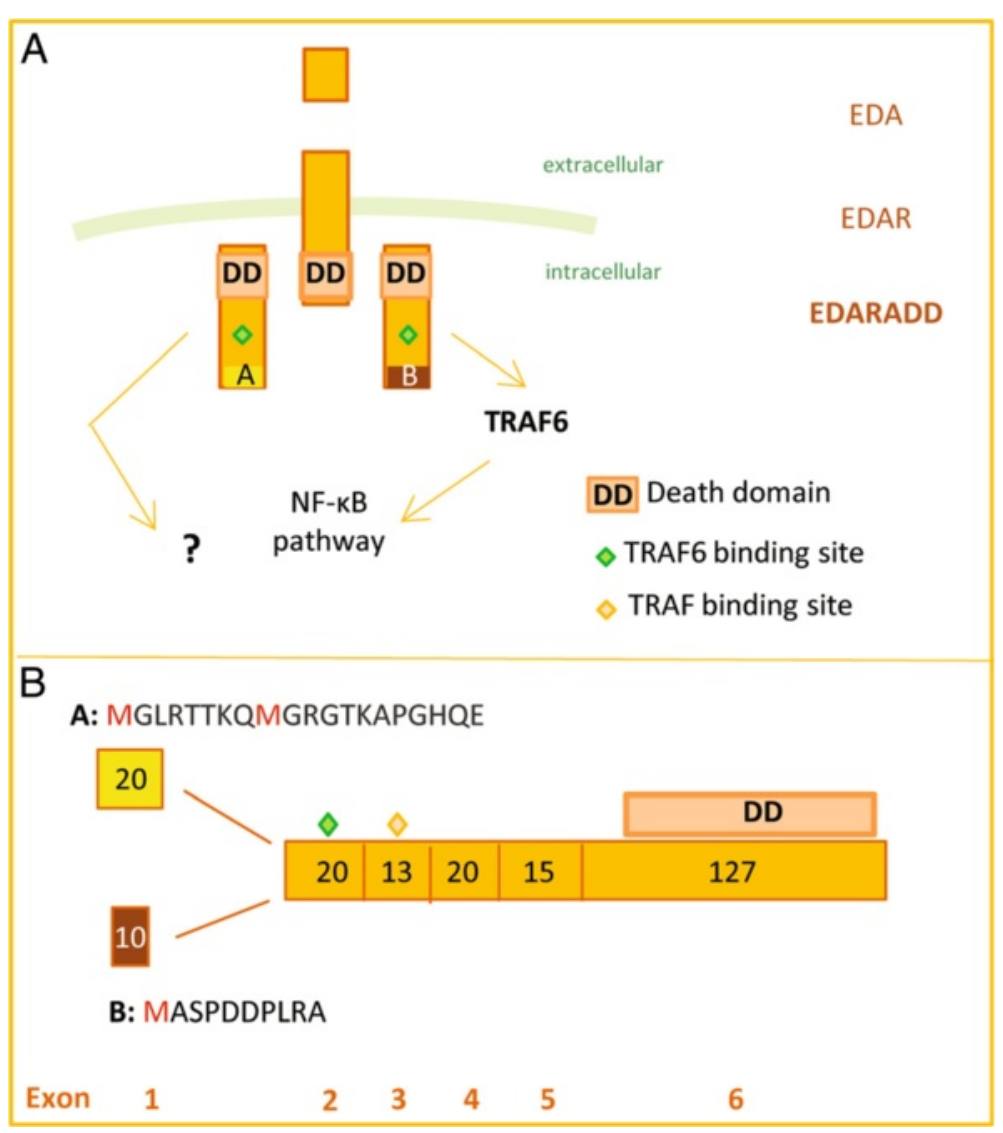

Fig. 1 The EDA pathway and its downstream signaling. a EDA binds EDAR that recruits EDARADD B by interacting with its death domain (DD), leading to the downstream activation of the NF-KB pathway [17]. EDARADD A displays the same functional domains as EDARADD B but its activity is not known. Proteins are shown here with a color code that highlights exon 1A/B boundary. $\mathbf{b}$ The two isoforms differ by their short N-terminal part corresponding to the first exon that is close to the TRAF binding sites. Proteins are represented with exon boundaries and exon size (in amino acid) is indicated. Sequences of the short $\mathrm{N}$-terminal peptide encoded by human exon $1 \mathrm{~A}$ and $1 \mathrm{~B}$ are represented in yellow (1A) and brown (1B)

had suggested that the two isoforms are likely expressed from two neighboring but different promoters. Therefore, besides their biochemical function, they could differ in terms of transcriptional regulation. Any of these differences could combine and translate in differential activation of the EDA pathway depending on the tissue and/or developmental time that could lead to phenotypic differences in terms of number and shape of ectodermal appendages. Based on published data, it is entirely possible that Edaradd isoforms differ in term of function and/or regulation, yet it remains to be proven.

When a new isoform is added to an old one during evolution, their ability to sustain phenotypic evolution in the long term might depend on the way the old and new isoform share the core gene function, that were ancestrally performed by the old isoform. Two extreme scenarios are possible: In the first, core functions are partitioned between both isoforms, in which case both may be strongly constrained. Alternatively, the ancestral isoform keeps core functions while the newly evolved isoform has more accessory roles, free to evolve in close association with phenotypic evolution. We expect that the gene will have a higher ability to support adaptive evolution (i.e. higher evolvability) in the latter case than in the former case, and in the former case than in absence of isoforms. Evaluating these possibilities for Edaradd not only requires a better knowledge of $\mathrm{A}$ and $\mathrm{B}$ isoform function, but firm conclusions about isoform $\mathrm{B}$ ancestrality and a broader view of $\mathrm{A}$ and $B$ isoform evolution in mammals.

Therefore, in this study, we questioned the evolution of $\mathrm{A}$ and $\mathrm{B}$ isoform in a broader set of mammals than done previously [18], associating in silico predictions and bench experiments to firmly conclude about functionality. We showed that B isoform is ancestral and conserved, while A isoform exhibits an unexpected mosaic conservation in mammals, with many independent losses found during their late diversification. Moreover, we dissected the respective biochemical function and regulation of the two isoforms, in a set of experiments performed in vitro, in cellulo and in vivo. We conclude 


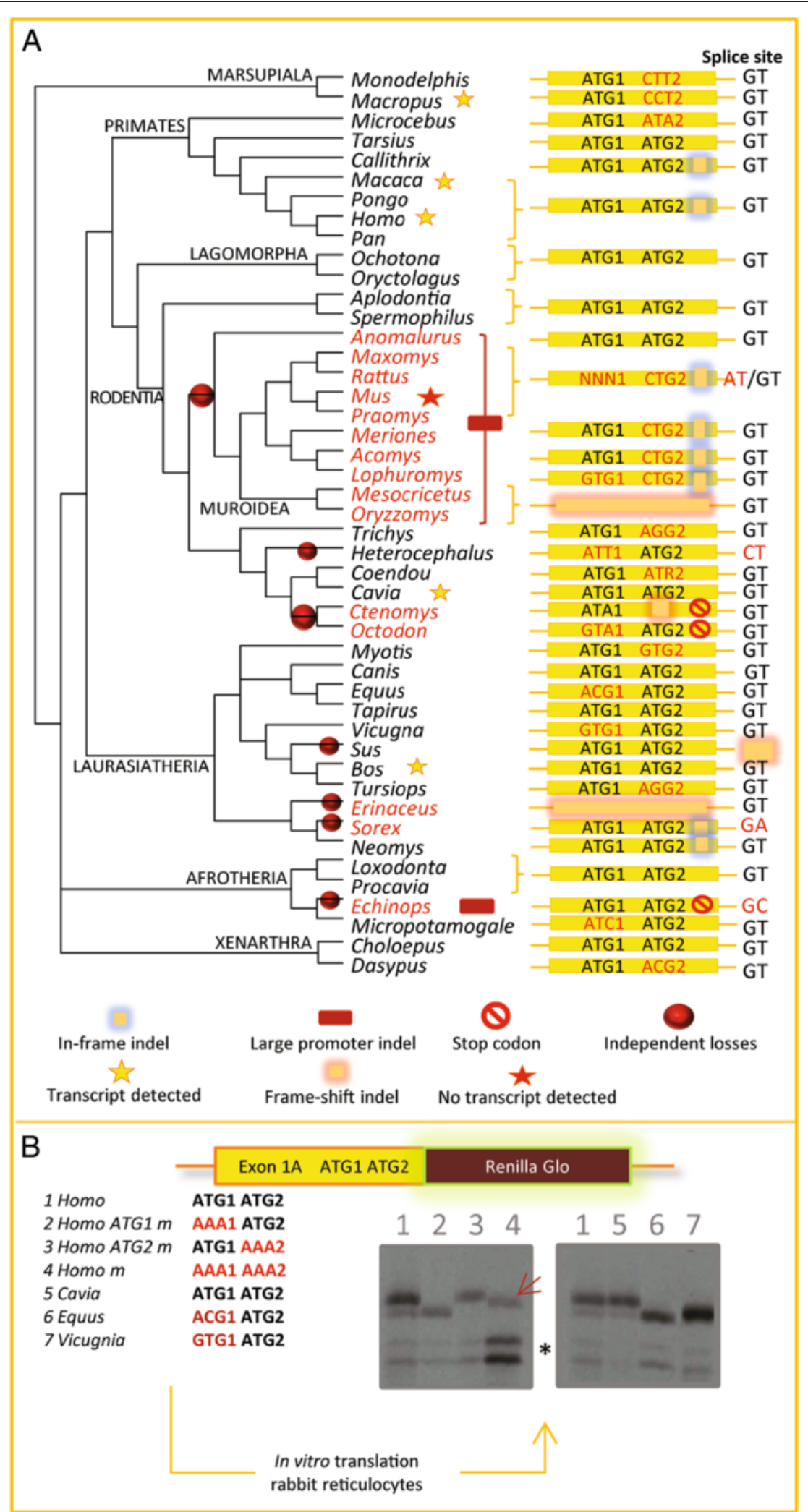

Fig. 2 (See legend on next page.) 
(See figure on previous page.)

Fig. 2 Mosaic conservation of the exon 1A in mammal evolution. a Examination of the exon 1A region in mammals. The yellow box represents the exon 1A in several mammal species [details in Additional file 6: dataset 56]. In human, two putative ATG could initiate the translation of a protein. For all species, these ATG are indicated as ATG1 and ATG2 when conserved. When not, the corresponding mutated codon is shown. The conservation of the promoter, the splice donor site and the frame were also studied and are indicated with symbols. The isoform-A transcript was detected by RT-PCR in human, guinea pig (Cavia) and wallaby (Macropus) or identified by BLAST against EST databases in macaca and cow (Bos). Species that are considered to have lost the ability to produce isoform A are shown in red. $\mathbf{b}$ In vitro translation experiments. The exon $1 \mathrm{~A}$ of Homo [43], Homo mutated for the first ATG (2), Homo mutated for the second ATG [44], Homo mutated for both ATG [43] Cavia (5), Equus (6), Vicugnia (7) were cloned upstream the Renilla gene to test by in vitro translation their ability to produce a protein from the first exon with different ATG 1 and 2 context. The asterisk shows two faint bands that are the result of low efficiency initiation at a downstream non-specific ATG. The dark red arrow points out the faint band initiated by an alternative AAG codon for the human double mutant

that the two isoforms mainly differentiated in term of regulation, keeping a similar function of NF-kB activation. We discuss how this combination of similar function and divergent regulation may enable differential regulation of the pathway in different developmental contexts, and we envision a scenario where the regulation of $\mathrm{A}$ isoform has been fast evolving in mammals in parallel with diversification of their ectodermal appendages, while B isoform was maintaining essential functions. We finally argue that isoforms generated from alternative promoters as exemplified by Edaradd could be an underappreciated mechanism ensuring the stability of some phenotypes and diversification of others during evolution.

\section{Results}

\section{The $B$ isoform of EDARADD is well conserved even outside mammals}

In order to enlarge our previous dataset [18], the genomic region of the exon $1 \mathrm{~B}$ was obtained by homology search (BLAST) against recently published genomes using the region of the closest available species as a probe. In previous work, we had suggested that the B isoform of EDARADD was homologous to the isoform found in chicken, suggesting that the $\mathrm{B}$ isoform could be the ancestral isoform whereas the A isoform would have been gained in mammals [18]. This finding was confirmed here by comparing sequences obtained from a larger panel of bird species (the extant vertebrate group closest to mammals). Indeed, both at the nucleotide and the amino-acid level, the N-terminal end of the mammalian $\mathrm{B}$ isoform exhibits strong similarities with the N-terminal end of the isoform found in birds [see Additional file 2: Figure S2]. In addition, the B isoform was found highly conserved in our upgraded set of 22 mammals [see Additional file 2: Figure S2]. More specifically, the promoter, the $5^{\prime} \mathrm{UTR}$ and the coding region of exon $1 \mathrm{~B}$ was found in all genomes, except in some low coverage genomes for which the genome assembly is obviously incomplete in this region.

\section{The $\mathrm{A}$ isoform has been repeatedly lost during late mammal diversification}

The picture is different for the $\mathrm{A}$ isoform. In previous work, we had found that the exon $1 \mathrm{~A}$ producing $\mathrm{A}$ isoform is conserved in a set of mammals but pseudogenized in mouse and rat [18]. This prompted us to increase our phylogenetic sampling by adding not only newly sequenced mammals (as done for B isoform) but also various rodent species, for which the region was obtained by PCR amplification followed by sequencing (Fig. 2A). We then aligned the putative promoter $1 \mathrm{~A}$ region found ca. $500 \mathrm{bp}$ upstream the ATG and looked for different criteria enabling functionality prediction, namely, the conservation of the promoter region, the splice donor site and the coding region with bona fide initiation site. In fact, in most species, exon 1A contains two ATG (here named ATG1 and ATG2) that can potentially initiate the translation: both are in frame and exhibit a canonical Kozak sequence, an indication of functionality.

However, a rapid look at this dataset revealed a very patchy conservation of ATG1 and ATG2: in some species (other than mouse and rat), ATG1 or ATG2 or both we mutated (Fig. 2A). Not only does such patchy conservation raise doubts as to whether a protein can actually be initiated in this exon $1 \mathrm{~A}$, but it is questionable whether a single ATG will be sufficient when the other is lost. Therefore, to be able to make realistic predictions from our dataset, we tested in vitro different constructs emanating from 4 different species where both ATG are present or one ATG is lost.

To this end, we cloned wild-type and mutant versions of the first exon of the $\mathrm{A}$ isoform (including the native 5 'UTR region known to influence translation efficiency) upstream of the Renilla reporter gene and performed in vitro translation (Fig. $2 \mathrm{~B}$ ). For the wild type human construct (lane 1), we detected two bands that are alternatively lost for the single ATG mutant constructs (lanes 2 and 3) and both lost in the double mutant construct (lane 4). This supports the view that the first (strong) band corresponds to initiation at ATG1 and the second (faint) band to a less efficient initiation at ATG2. In such a test, it is common to observe that the presence of a potent ATG strongly limits initiation at downstream or nonspecific initiation sites, as ribosomes will preferentially initiate at the first site that they encounter during ribosomal scanning. However, if the most $5^{\prime}$ ATG is 
mutated, then initiation can occur with a higher frequency at these other sites. This also explains why other bands are observed in the double mutant (lane 4). Indeed, we observed two strong much lower bands (asterix, lane 4) that likely correspond to initiation at downstream sites, found within the renilla sequence (note that these two same bands are accordingly also hardly detected with all other constructs: see lanes 1 to 7). We also observed one faint band (lane 4, dark red arrow), which is slightly lower than the one seen with the wild type construct (compare lane 4 with lanes 1 and $3)$. Here, an analysis of the sequence showed the presence of a "near cognate AAU" site (an AAG codon), which is located 3 codons downstream from the ATG1 and was likely recognized by ribosomes, producing this faint band. With the guinea pig construct containing both ATG1 and ATG2, we obtained a similar pattern of initiation as with the wild type human construct (lane 5). Finally, in two species where only the ATG2 remains (Equus and Vicugna), initiation efficiently occurred at this site, as it is the case with the human ATG1 mutant construct (lane 6 and 7). We concluded that ATG1, or ATG2 (when ATG1 is missing), are potent initiation sites. As a consequence, we then infer in Fig. 2A that the A isoform is lost only when the two ATGs are mutated and/or the promoter region or the splice donor is mutated or absent (species indicated in red in Fig. 2A).

Our analysis revealed that in muroid rodents, the promoter region is completely rearranged: we observed a deletion of the conserved region where the transcriptional start site is mapped in human, replaced by a $72 \mathrm{bp}$ insertion (see the red brace encompassing muroid rodents and the red box symbolizing the deletion in Fig. 2A). Mosaic deleterious events are observed in the coding sequence (ATG1 and/or ATG2 lost, frame-shift indels). This pattern strongly suggests that a deletion of the promoter region occurred in the common ancestor of muroids and abrogated the transcription of exon $1 \mathrm{~A}$ (as observed in mouse, where the pseudogenized exon $1 \mathrm{~A}$ is not transcribed, [18]). Following this event, constraints on the coding sequence were relaxed, leading to its mosaic degeneration in muroid rodents.

In other mammals, we found other losses (Fig. 2A). In Ctenomys and Octodon, two rodents that are more closely related to guinea pig where the $\mathrm{A}$ isoform is expressed [18], frame-shift indel and stop codons are observed. In Echinops, the promoter region exhibits a deletion and a stop codon is found in the coding sequence. The whole region is lost in Erinaceus and the splice site is lost in Sus as well as in Sorex. Because these species belong to very different branches of the mammalian tree or the mutation events are obviously independent, we could map at least 7 independent losses (red points in Fig. 2A). It is noteworthy that isoform-A is found in marsupials and in all main groups of placental mammals. Losses occurred later, during the diversification of these groups, as exemplified in rodents. In the next sections, we compared the function and regulation of $\mathrm{A}$ and $\mathrm{B}$ isoforms.

\section{The two EDARADD isoforms are expressed by two alternative promoters that are differentially regulated}

The existence of two distinct Edaradd mRNA [18] differing by their first exon suggests that two alternative promoters could control the expression of the two isoforms. To gain insight into the possible proximal regulation of the expression of both isoforms, we cloned the $5^{\prime}$ regions of the two putative promoters from two representative mammalian species upstream of a luciferase reporter gene and tested them in HEK cells that expressed both isoforms ([19] and data not shown). We chose the human gene which has the two isoforms, and the mouse which has lost the A isoform (Fig. 3A). When transfected in HEK cells, the B promoter exhibited a strong activity that was conserved in human and mouse (Fig. 3C). In contrast, the A promoter exhibited a low but significant activity (Fig. 3B). Because signaling pathways often feedback either positively or negatively [2], we tested promoter response to the EDA pathway activation by co-transfecting EDAR that induces an overactivation of the pathway in cellulo [20] (Fig. 3D and E). Whereas the A promoter did not exhibit any variation of its activity compared to the empty vector, the B promoter was downregulated in a dose-dependent manner by growing amount of transfected EDAR. This downregulation was observed with other NF- $\mathrm{KB}$ activators such as TRAF6 and partially rescued by NF- $\mathrm{kB}$ downregulators such as CYLD (Fig. 3F). It has to be noted that no cell death was detected during experiments (data not shown). Taken together, these results show a different regulation of the two promoters depending on the cellular context. To further investigate differences in transcriptional regulation in vivo, we studied the expression of the two isoforms in different tissues from two placental mammals (human, guinea pig - Cavia porcellus in Fig. 2A) and a marsupial (tammar wallaby, Macropus eugenii in Fig. 2A) by RT-PCR experiments and in human cell lines using the ENCODE data (Fig. 3G). The two isoforms were generally co-expressed in tissues and cell lines except in human skin and brain and in embryonic cell lines in which only the B isoform was detected. When co-expressed, the two isoforms were either expressed at the same level or the B isoform was more strongly expressed than the A isoform. This shows that the transcripts of A and B isoforms are differentially regulated in vivo and in cellulo in line with the differential regulation of their promoters in luciferase assays. 


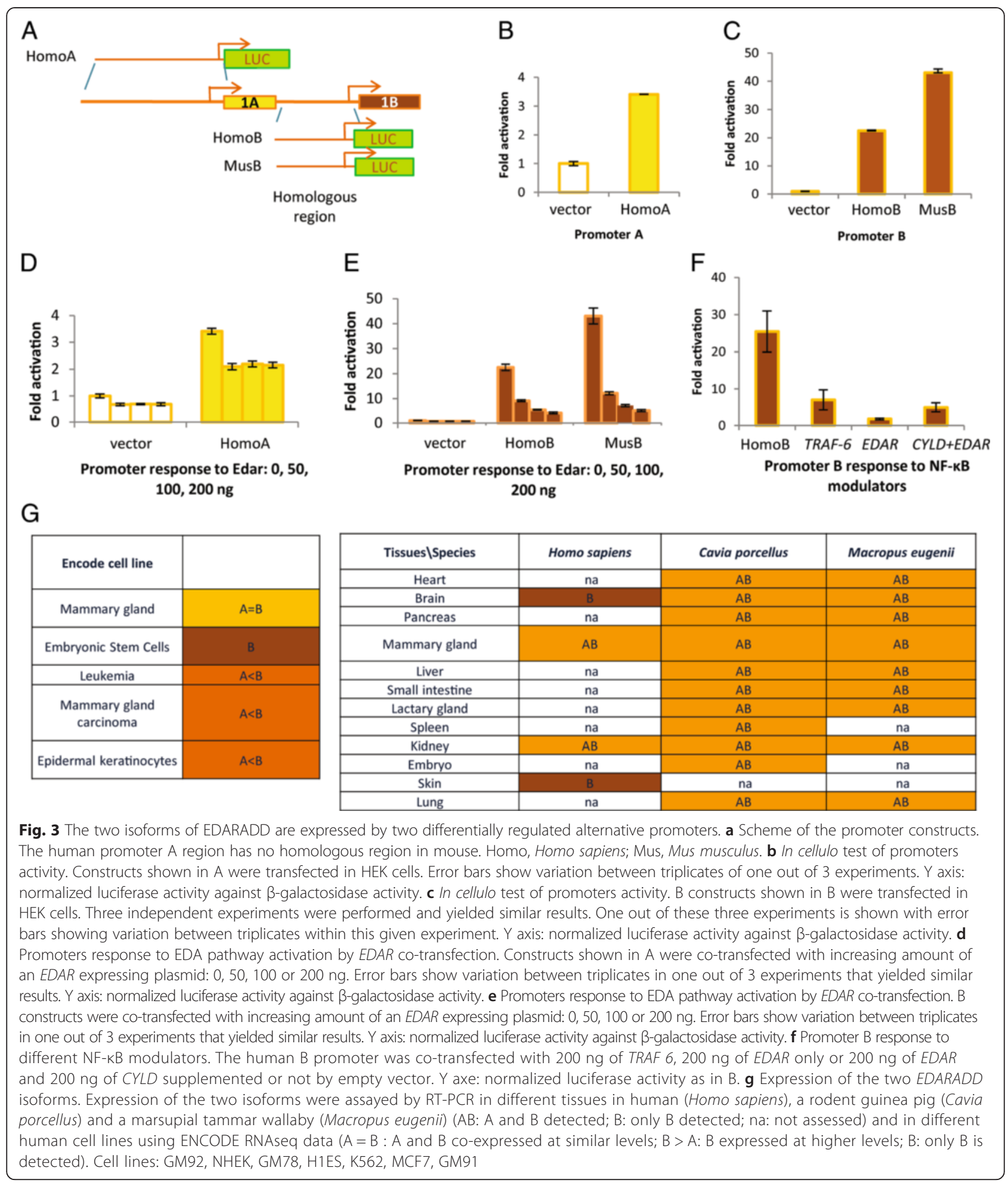

The human A isoform of EDARADD is more active than the $B$ isoform but accumulates more slowly

To decipher the biochemical role of the two EDARADD isoforms, we studied the function of the two human proteins in cellulo (Fig. 4) using the same HEK cells. We used recombination methods to generate expression plasmids for the two proteins that are rigorously identical (Fig. 4A), except for the small N-terminal peptide characterizing the A or B isoform (notably, translation initiation sites were identical). Since the first known 


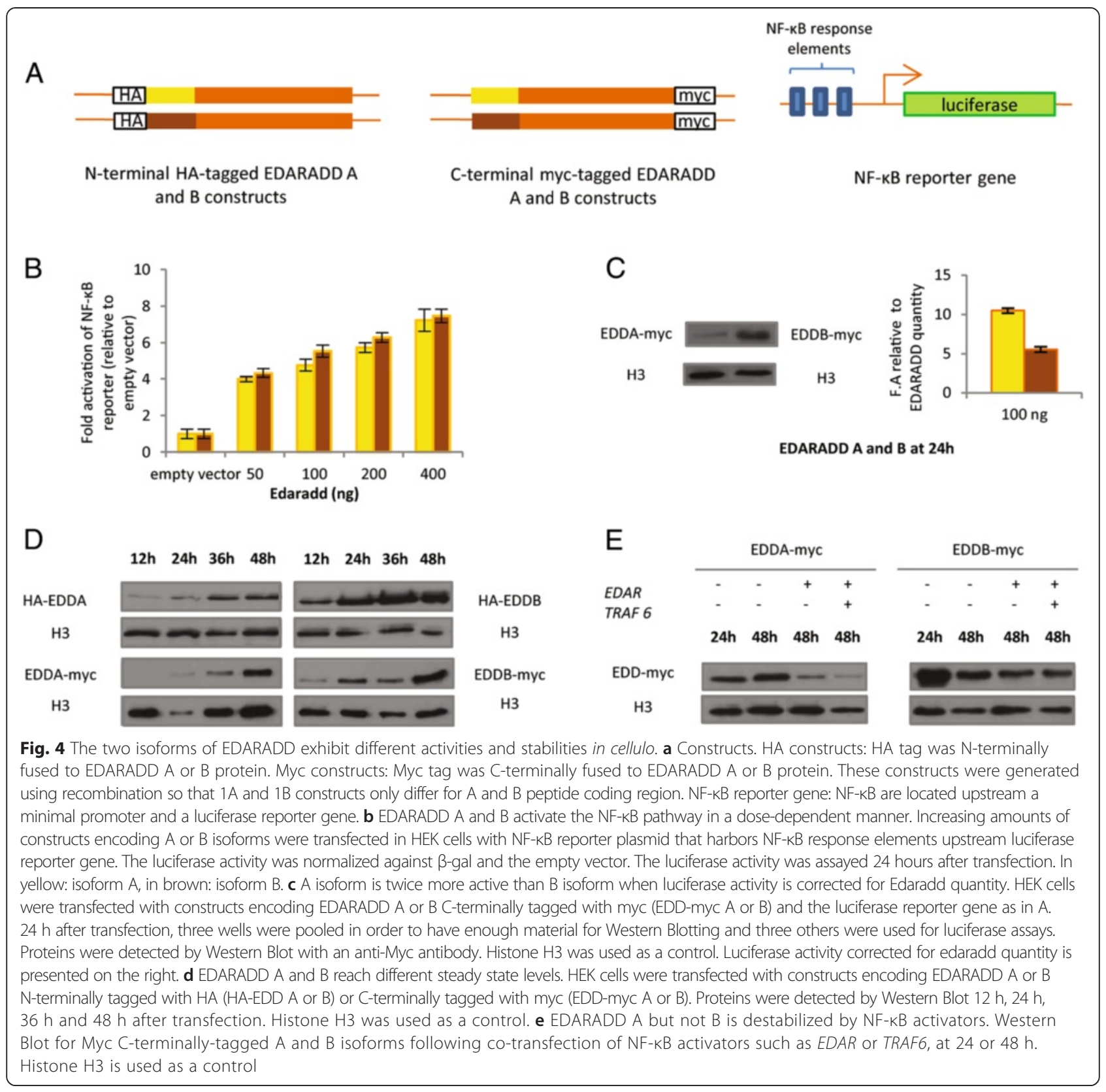

function of the EDA pathway is to activate the NF- $\mathrm{kB}$ pathway we tested the ability of myc tagged-versions of both isoforms to activate the NF- $\kappa B$ pathway in a NF- $\kappa B$ reporter luciferase assay. As previously described, the $\mathrm{B}$ isoform activated the NF- $\mathrm{kB}$ pathway reporter in a dosedependent manner [19] (Fig. 4B). The A isoform also activated the NF-kB pathway reporter in a dose-dependent manner. Examination of the protein level by western blot showed that the amount of $\mathrm{B}$ isoform was 2.5 higher than the amount of $\mathrm{A}$ isoform (Fig. $4 \mathrm{C}$ and $\mathrm{D}$ ). Similar results were obtained whenever an $\mathrm{N}$-terminal or a C-terminal tagged version of the proteins was used. Thus, to compare the activity of both isoforms, the luciferase activity needed to be corrected by the amount of EDARADD protein detected in the lysates. In so doing, the $\mathrm{A}$ isoform appeared to be twice more active than the B isoform (Fig. 4C). The co-transfection of the two constructs together at different concentrations did not exhibit any synergistic nor antagonistic effect [see Additional file 3: Figure S3]. We then followed the accumulation of the two proteins for 48 hours and found that the B isoform accumulated more rapidly than the A isoform (Fig. 4D). Considering the steady-state level of the two proteins, at 48 hours, the $\mathrm{A}$ isoform disappeared when co-transfected with EDAR or other NF- $k \mathrm{~B}$ activators (Fig. 4E) whereas the $B$ isoform remained stable 
suggesting a difference of regulation at the protein level. Similar results were obtained in presence of a proteasome inhibitor (MG132), presumably ruling out proteasome-dependent degradation (data not shown). In summary, the two human isoforms of EDARADD exhibit different functionalities in cellulo: they both activate the NF-kB pathway but exhibit differences in their dynamics: the $B$ isoform accumulates more rapidly whereas the A isoform is more active but downregulated at the protein level following pathway activation. Such differences could impact their in vivo functions in transducing the NF- $\mathrm{kB}$ pathway both quantitatively and qualitatively.

The two human isoforms of EDARADD exhibit different activities in a rescue experiment performed in zebrafish We next used the zebrafish system to assess if the two isoforms exhibited functional differences. To this end, we performed a rescue experiment where endogenous edaradd function has been obliterated by morpholino injections but is complemented by isoform A or B mRNA injections (Fig. 5). It is known that alterations of

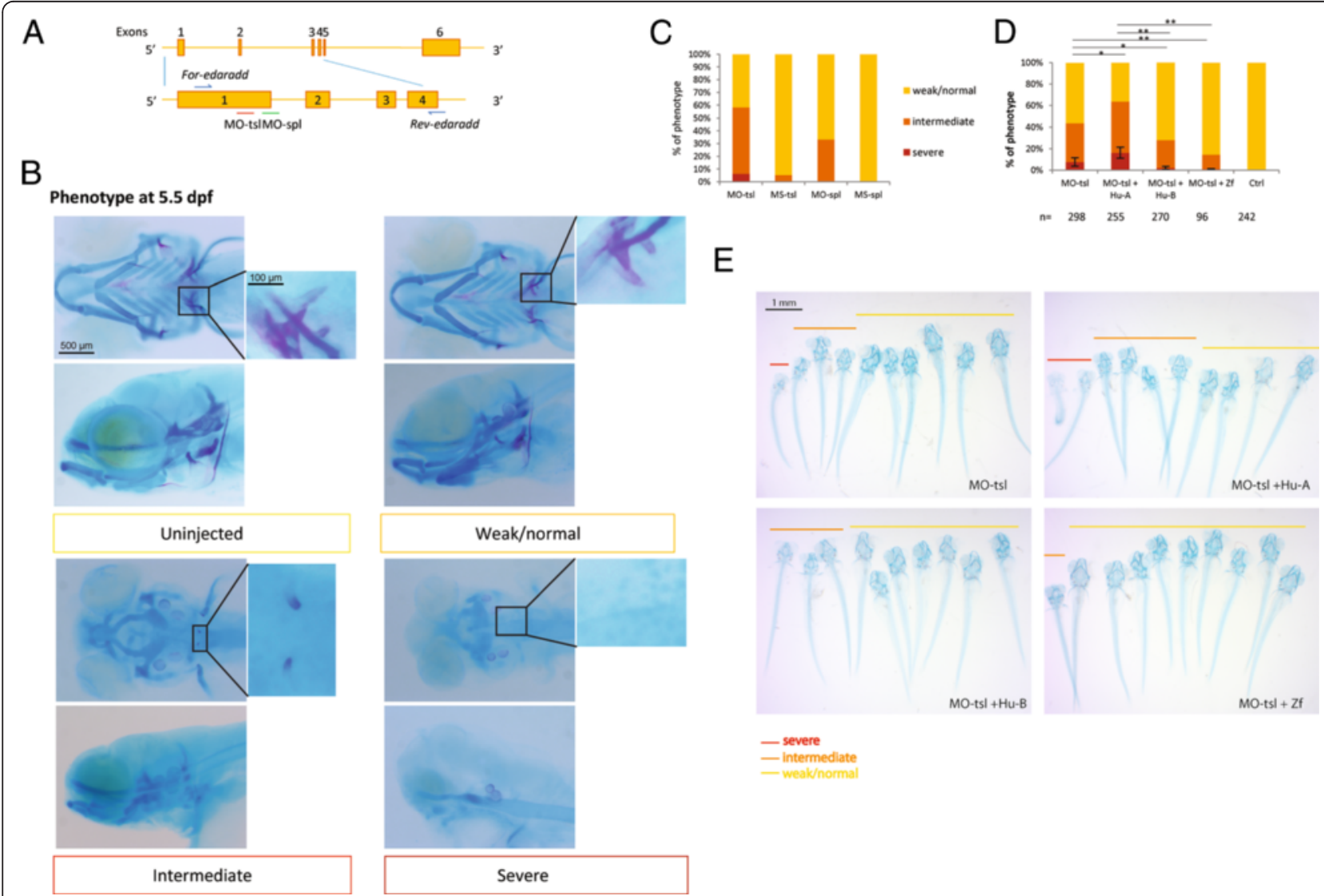

Fig. 5 Human EDARADD B but not EDARADD A rescues skeletal defects observed in MO-injected zebrafish larvae. a Schematic representation of the exon-intron structure of the zebrafish edaradd gene. The location of target sequences for translation-blocking (MO-tsl) and splice-blocking (MO-spl) edaradd morpholinos is indicated by a red and a green line, respectively. Blue arrows indicate the location of the primers used for edaradd mRNA expression analysis during development [see Additional file 4: Figure S4] and for splice-blocking edaradd MO validation by RT-PCR analysis [see Additional file 4: Figure 54]. b Knockdown of Edaradd expression leads to skeletal defects. WT embryos were injected at one-cell stage and skeletal morphogenesis was analyzed through alcian blue and alizarin red staining at $5.5 \mathrm{dpf}$. The larvae phenotypes observed were classified as severe, intermediate or weak/ normal phenotype. The severe phenotype presented no pharyngeal skeleton, a rudimentary neurocranial cartilage and no tooth could be detected. This defect was also accompanied by pericardial and yolk sac edema. Intermediate phenotype showed rudimentary pharyngeal skeleton without second arch and gill arch derivatives and sometimes one rudimentary tooth on each side of the larvae. Weak/normal phenotype demonstrated small gill arch derivatives or no obvious defects and at least one tooth on each side of the larvae could be clearly observed through alizarin red staining. Ventral and lateral views of the head are shown at low magnification (scale bar: $500 \mu \mathrm{m}$ ) and a zoom on the teeth is shown on the right panel (scale bar: $100 \mu \mathrm{m}$ ). c Percentages of the various phenotypic groups obtained following MO injection [see Additional file 4: Figure S4] or MS (Mismatch control) injection. These results are representative of at least 3 independent experiments following injection of at least 70 embryos per condition. $\mathbf{d}$ Human EDARADD A and B mRNA effects in rescuing experiments. One-cell stage embryos were co-injected with MO-tsl and/or capped human EDARADD A or B, or capped zebrafish edaradd mRNA with at least 70 embryos injected per condition. The barplot shows the percentage of each phenotypic group in 4 separate experiments and the statistical analyses have been performed on the severe group. e A representative group of 10 larvae, classified according to their phenotype, are shown for each condition 
EDA pathway in zebrafish result in abnormal tooth and dermal skeleton development [21], but little is known about edaradd during early zebrafish development, i.e. the developmental window mainly targeted by morpholino injections.

Therefore, to help the interpretation of these experiments, we first gathered expression data for the endogenous unique splice variant of edaradd gene during zebrafish development. By semi-quantitative RT-PCR, we observed a high maternal expression of edaradd, which rapidly decreased at $8 \mathrm{hpf}$ (hours post fertilization). edaradd expression then slowly increased from $12 \mathrm{hpf}$ to 96 hpf [See Additional file 4: Figure S4]. By in situ hybridization, we could not detect a regionalized edaradd expression pattern (possibly due to low expression levels) [Additional file 5: Figure S5] but both eda and edar were expressed in the pharyngeal region from 48 and 24 hpf respectively, suggesting that the EDA pathway (and therefore edaradd) was active at least in this region during this period of zebrafish development.

We next investigated the function of edaradd gene during zebrafish embryonic development using morpholino antisense oligonucleotides directed either against edaradd translation initiation site (MO-tsl) to disrupt the translation of the protein or against edaradd exon 1 splice donor site to disrupt mRNA splicing (MO-spl) (Fig. 5A). Splice MO efficiency was confirmed by RTPCR [see Aditional file 4: Figure S4 for details]. The edaradd MO-injected larvae exhibited abnormalities of the pharyngeal and cranial skeleton visible at $5.5 \mathrm{dpf}$ (days post fertilization) following cartilage and mineralized tissue staining (Fig. 5B and C), consistent with the expression pattern evidenced for eda and edar. These embryos were classified depending on the severity of the skeletal defects and on the presence of teeth (see Fig. 5B for a detailed description). MO-tsl and MO-spl-injected larvae led to similar cartilage defects except that MO-spl had weaker effects, even at high concentrations [see Additional file 4: Figure S4].

To study the in vivo activities of the two human EDARADD isoforms, we tested their ability to rescue the phenotype of the edaradd morphants. We first checked that injection of EDARADD $A$ or $B$ mRNA or zebrafish edaradd in wild-type embryos had no obvious effect even following injection of $100 \mathrm{pg}$ of mRNA per egg [see Additional file 4: Figure S4]. In particular, no supernumerary teeth were detected when observed at 5.5 dpf. We then co-injected 25 to 100 pg human EDAR$A D D A$ or $B$ or zebrafish edaradd mRNA with MO-tsl in WT embryos (Fig. 5D and E) and analysed the corresponding larvae as above. We first confirmed that zebrafish edaradd mRNA efficiently rescued the MO-tsl-induced skeletal defects. Human EDARADD B mRNA also provided significant rescue $(2.1 \pm 1.5 \%$ larvae corresponding to the severe phenotype for MO-tsl + EDARADD $B$ mRNAinjected embryos compared to $7.6 \pm 3.9 \%$ for MO-tslinjected embryos $(p=0.039))$. In sharp contrast, EDARADD A mRNA significantly increased the defects observed in MO-tsl-injected embryos $(16.3 \pm 5.1 \%$ larvae corresponding to the severe phenotype for MO-tsl +EDARADD A mRNA-injected embryos compared to $7.6 \pm 3.9 \%$ for MOtsl-injected embryos $(p=0.035))$. Importantly, both the rescue and the worsening phenotypes were sensitive to the dose of EDARADD mRNA injected, with 50 and $100 \mathrm{pg}$ of $E D A R A D D$ mRNA producing significant and equivalent effects whereas $25 \mathrm{pg} E D A R A D D$ mRNA leading only to slight effects (data not shown). Taken together these data show that the two human isoforms are not functionally equivalent in this heterologous system.

\section{Discussion}

In this case study focused on the two alternative isoforms of the EDARADD adaptor, we investigated how a signaling pathway important for morphological evolution [22] could evolve functionally by gain and loss of protein isoforms. This let us characterize the evolution but also the function of EDARADD A and B isoforms. As some of our results are of particular interest with respect to the EDA pathway's role in development and Human Ectodermal Dysplasia (HED) disease [23-26]) we first review and discuss in some detail the interpretation of our biochemical experiments, before returning to on our main focus, which is to integrate the later results with results about isoform evolution.

\section{EDARADD isoforms: activity and regulation}

Because previously little was known about the functional role of EDARADD A isoform, we compared the activity of both human isoforms in cellulo, using the same cell lines (HEK cells) as previous studies [19, 20, 23]. HEK cells are kidney epithelial cells. Even if the EDA pathway is mainly known for its role in ectodermal cells, EDA pathway genes are indeed expressed in the kidney (as well as in other epitheliae without an ectodermal origin, e.g. lung, intestine) and Edaradd in particular is detected in HEK cells [19]. We focused on the activation of the NF- $\mathrm{kB}$ pathway as this is the major pathway activated by EDA [22]. We showed that the A isoform is more active than the $\mathrm{B}$ isoform as, for the same quantity of protein, the activation of the NF- $\mathrm{kB}$ pathway is 2.5 times higher in a reporter luciferase assay. It is not surprising that both isoforms are capable of activating the NF- $k B$ pathway: beside their $\mathrm{N}$-terminal difference, they share the same functional domains, such as the TRAF binding sites or the death domain interacting with EDAR (Fig. 1). The N-terminal part of proteins is often implicated in protein folding and stability [27], and this might explain two differences observed between the $\mathrm{A}$ and $\mathrm{B}$ proteins. 
First, the very $\mathrm{N}$-terminal peptide could influence the folding of the N-terminal part, which may then be specific to each isoform. Since this N-terminal part contains the binding site for TRAF6 (Fig. 1B), a major interactor of EDARADD in transducing downstream signaling [27], a difference in folding may easily turn into the observed difference in level of activation of the NF- $\mathrm{KB}$ pathway. Second, the N-terminal peptide could affect the protein stability and explain the difference in steady-state levels. Linked to this, we showed that the A isoform is downregulated by the activation of the pathway, in contrast to the $\mathrm{B}$ isoform. This downregulation of the A protein level is linked to the activation of the pathway as the addition of TRAF 6 further enhances the decrease in protein level. The mechanism for this downregulation is unclear, but does not seem to involve proteasomedependent degradation. Together, these results obtained in an overexpression system suggested that the human A and B EDARADD proteins are biochemically different, despite that they differ only in a very short $\mathrm{N}$-terminal peptide. However, this difference may impact EDA pathway activation quantitatively rather than qualitatively.

The rescue experiments performed in vivo in zebrafish further confirmed this biochemical difference. Before performing this rescue, we characterized the effects on zebrafish development of edaradd downregulation by morpholino injection. The latter resulted in defects of pharyngeal tooth formation, which could be expected based on the phenotype observed in teeth of other fishes [21, 28] and mammals [22]. However, the craniofacial skeleton was also abnormal. This was in line with eda and edar expression in the developing craniofacial region detected from 48 and $24 \mathrm{hpf}$ respectively. Interestingly, both in mouse and human, a mutation in the Eda gene leading to defects in the craniofacial skeleton has also been observed [29]. Our results thus highlight an underappreciated role of the EDA pathway in craniofacial development. We then tested the ability of human A and $\mathrm{B}$ isoforms to rescue this phenotype. The human $\mathrm{B}$ isoform, but not the A isoform, is capable to rescue the edaradd MO-induced phenotype. Of note, this rescue observed despite EDARADD B overexpression has no major effect on zebrafish development. This is reminiscent of the results with the K14-Edaradd mice, which overexpress Edaradd in skin appendages. These mice display no overt phenotype, yet the transgene can rescue the mutant hair follicle and tail kinking phenotype ([30, 31] and D. Headon, personal communication). This rescue experiment suggests that the $B$ isoform is functionally closer to the single zebrafish Edaradd protein and thus probably closer to the ancestral EDARADD protein predating $\mathrm{A}$ isoform gain in mammals. In contrast, the human isoform $\mathrm{A}$ is unable to rescue the edaradd MO-induced phenotype. Worse, it tends to enhance phenotype severity. This suggests that the A protein may exert a dominant negative effect on the remaining endogenous Edaradd protein. The human A isoform could compete with the endogenous protein, but being unable to transduce downstream signaling in zebrafish. Alternatively, as suggested in cellulo, the human A isoform could be rapidly destabilized following pathway activation and in turn destabilize the endogenous protein through heteromer formation. Whatever the explanation, these experiments showed that the two isoforms are functionally different in vivo. These differences will have to be further explored in vivo in mammals. For example, in a near future, genome-engineering strategies could in principle be applied to species that possess both isoforms such as guinea pig.

Finally, we also studied the transcriptional regulation of the two isoforms. We found that the A promoter exhibits a weak activity in HEK cells whereas the B promoter is more active and exhibits a feedback regulation following activation of the pathway. Moreover, the B isoform is expressed in all tissues and cell lines checked in this study. In contrast, the A isoform is more often expressed at lower levels in cell lines and is not found in some tissues and cell lines. This suggests that the B promoter may drive $B$ isoform expression in all epithelial tissues making use of the pathway, but be turned off upon activation of the pathway, whereas the A promoter may confer to the A isoform a more tissue-restricted expression, which remains unchanged (at the transcriptional level) when the pathway is activated.

Putting together the transcriptional and posttranscriptional levels, there are clear regulatory differences between the two isoforms despite the fact that both activate the NF- $K B$ pathway. The $B$ isoform is well expressed, stable, activates NF- $\mathrm{kB}$ consistently and exhibits feedback regulation at the transcriptional level. The A isoform is expressed in more specific contexts, activates NF- $\mathrm{kB}$ strongly but is downregulated by a unknown feedback mechanism at the protein level. Thus it appears that the two isoforms should enable regulating the EDA pathway in a tissue-specific manner and in different ways, in terms of level of activation and dynamics. In the context of the development of ectodermal appendages, [22, 32], such flexibility of the pathway activation is interesting, as the level and dynamics of the pathway activation is expected to have phenotypic consequences on the patterning of ectodermal appendages. Indeed, we know from many examples of experimental tinkering of the pathway that changes in activity translate into phenotypic changes in terms of number, size and shape of ectodermal appendages (e.g. in hair [33] and teeth [34]), some highly resembling natural species variation $[35,36]$. 


\section{A case study for the evolutionary tinkering of signaling pathways by isoforms evolution}

Previously, the expansion of signaling pathways repertoire was mainly explained by gene or genome duplication [1] but recent transcriptomic studies revealing the ubiquity and lability of alternative isoforms [9] suggest to add the evolution of isoforms as a key mechanism. EDARADD nicely exemplifies this notion.

We showed that the B isoform is the ancestral isoform and is conserved in all examined species. Several results suggest that the B isoform has kept the core, vertebrateconserved, functions of EDARADD: i) it is highly similar at the amino-acid level to the single isoform found in birds and even in fish ii) the human B isoform is able to rescue the zebrafish morphant, iii) it is expressed in all tissues where the pathway is found. In sharp contrast, the $\mathrm{A}$ isoform newly evolved in mammals due to a new upstream promoter and was repeatedly lost during mammal diversification. Its expression pattern suggests a more accessory role than isoform $\mathrm{B}$. Despite that it also activates the NF- $\mathrm{B}$ pathway as isoform $\mathrm{B}$, it turned out that it clearly differs from isoform B in terms of transcriptional and post-translational regulation. Therefore, our results suggest that this new A isoform brought new possibilities and levels of regulation to the EDA pathway, different from B isoform. Moreover, our results highlight the highly mosaic albeit nonrandom evolution of A isoform. Indeed, A isoform is present in both marsupial and placental mammals (Fig. 2, see also Fig. 4 for expression) that diverged 165-215 MYA [37], and in most groups of placental mammals. This contrasts with the high rate of losses found during late (post- KT boundary) mammals diversification. This suggests that the loss of isoform $\mathrm{A}$ is specifically associated with mammal diversification and the underlying extensive diversification of their ectodermal appendages (hair, teeth, glands, nails...) in terms of number and shape. It is not easy to find a common phenotypic trend in the lists of species where A isoform is lost. Although we may have missed it, we rather favor the possibility that the independent losses of $\mathrm{A}$ isoform are associated with a number of different phenotypic changes. Indeed, it is likely that isoform A regulation evolved rapidly along the mammalian tree together with changes in ectodermal appendages, the loss in some species being just extreme cases in which the regulation diverged up to be lost. The loss could occur either because changes in ectodermal appendages phenotype made it dispensable or because the loss participated in reaching an adaptive phenotype.

\section{Conclusions}

In summary, as the EDA pathway is a hot spot of adaptation [22], it is easy to imagine that by modulating the level of activation of the EDA pathway through space and time, the regulation of these two isoforms (and especially A) could follow or sometimes allow rapid evolution of ectodermal appendages. Simultaneously, the two isoforms (and especially B) could ensure that essential functions are maintained. If true, this duality between an ancestral slowly evolving isoform and a newly rapidly evolving isoform may provide a strong evolvability to the pathway in terms of spatio-temporal regulation, while ensuring that essential functions are maintained. This case study suggests we pay greater attention to mosaic evolution of isoforms as an important mechanism of phenotypic change.

\section{Methods}

\section{Exon $1 \mathrm{~A}$ region sequence}

The exon 1A region of 24 Mammals (21 rodents, three nonrodent) was obtained by extracting total genomic DNA from ethanol-preserved tissues obtained from the collection of Preserved Mammalian Tissues of the Institut des Sciences de l'Evolution of Montpellier (France) with a DNeasy Blood and Tissue extraction kit (Qiagen). The region was then PCR amplified and sequenced twice independently using primers F4 AGAARGAACCACARACCAAACCTC and R4 TTTGGCATTAGTTACTGCCTGACC. Other mammal species sequences were obtained by tBLASTN on Ensembl genomes and TRACE release 59 (3 August 2010) accession number are in the Additionnal file 6: Dataset S6. Sequences were aligned using Muscle (http://www.drive5.$\mathrm{com} / \mathrm{muscle} /$ ) [38] followed by manual refinements and visualized in Seaview (http://pbil.univ-lyon1.fr/software/seaview.html). [See dataset in Additional file 6: Dataset S6].

\section{in vitro translation experiments}

Eight exon 1A constructs (Human, Human ATG1 mutant, Human ATG2 mutant, Human double mutant, Cavia porcellus, Equus caballus, Meriones crassus, Vicugnia pacos) were synthetized (the limits of exon $1 \mathrm{~A}$ in the different species being defined by sequence homology to the human exon $1 \mathrm{~A})$, cloned in poRenilla vector and tested by in vitro translation as [39]. Briefly, p0Renilla-derived vectors containing a 100 nt long poly(A) tail were linearized by EcoRI digestion. Capped mRNAs were transcribed in vitro $2 \mathrm{~h}$ at $37^{\circ} \mathrm{C}$ using $1 \mu \mathrm{g}$ of linear DNA template; $80 \mathrm{U}$ of T7 RNA polymerase (Promega); 40U of RNAsin (Promega); $10 \mathrm{mM}$ of rCTP, rUTP, rATP; 0.36 mM rGTP; 30 mM DTT; $1.24 \mathrm{mM}$ of $\mathrm{m}^{7} \mathrm{GpppG}$ cap analogue (New England Biolabs) in transcription buffer (40 mM Tris- $\mathrm{HCl}(\mathrm{pH} 7.9), 6 \mathrm{mM} \mathrm{MgCl}_{2}$, $2 \mathrm{mM}$ spermidine and $10 \mathrm{mM} \mathrm{NaCl}$ ). mRNAs were DNase treated and precipitated with 1 volume of ammonium acetate $5 \mathrm{M}(2.5 \mathrm{M}$ final concentration). The integrity of mRNAs was checked on agarose gel electrophoresis and their concentration was quantified by spectrophotometry at $260 \mathrm{~nm}$ using Nanodrop (NanoDrop Technologies, Wilmington, 
DE, USA). Then, in vitro transcribed RNAs were translated for $30 \mathrm{~min}$ at $30^{\circ} \mathrm{C}$ in $10 \mu \mathrm{l}$ of the supplemented untreated RRL or the Flexi Rabbit Reticulocyte System 50 \% (v/v) each (Promega Co., Madison, WI, USA) in the presence of $\mathrm{KCl}$ (75 mM), $\mathrm{MgCl}_{2}(0.5 \mathrm{mM}), 20 \mu \mathrm{M}$ of amino acids mix minus methionine and $0.6 \mu \mathrm{Ci}$ of $\left[{ }^{35} \mathrm{~S}\right]$-methionine (GE Healthcare Life Sciences). Reactions were stopped with $2 \times$ SDS-loading buffer and the products were resolved by $15 \%$ SDS-PAGE. Gels were dried and subjected to autoradiography using Biomax films (Eastman Kodak Co.).

\section{Promoter analysis}

We amplified by PCR the $5^{\prime}$ region of exon $1 \mathrm{~A}$ in human and exon 1B in human and mouse. We took 2900 $\mathrm{pb}$ upstream the ATG of the exon 1A for the human A promoter and the entire region between the end of the exon $1 \mathrm{~A}$ and the ATG of the exon $1 \mathrm{~B}$ for the human $\mathrm{B}$ and mouse $B$ promoters. We used: for human $A$ promoter: FhumA 5' - TGGCACATTCTGAGGCATAG-3', RhumA 5' - AAGATGCTCGCTGGTTGTCT-3'; for human B promoter: FhumB 5'-ATATGCTAGCGCATT TGCTGTCTTCCTGG-3', RhumB 5'-GAGACTCGAG GGCAAACGAGCGTCCG-3', for mouse B promoter: FmusB 5' - ATATGAGCTCAACTCCAGACTCCCCTC CT-3', RmusB 5'-ATATCTCGAGCCCTGAGAACAG CAGGAT-3'.

These fragments were cloned into pGL2 basic vector upstream luciferase reporter gene to test the ability of the two putative promoters to initiate the transcription in HEK cells. HEK cells grown in 24-well plates were then transfected using Exgen500 transfection reagent (Euromedex). Each well received $400 \mathrm{ng}$ of each promoter cloned in pGL2basic vector and $100 \mathrm{ng}$ of $\beta$-galactosidase expression plasmid. For the tests with putative activators such as EDAR, TRAF6 or CYLD, promoter vectors were cotransfected with i) growing amount of EDAR 50, 100 or $200 \mathrm{ng}$ supplemented by empty pCDNA3, or $200 \mathrm{ng}$ of EDAR cloned into pCDNA3, or $200 \mathrm{ng}$ of TRAF6 cloned into pCDNA3 or $200 \mathrm{ng}$ of EDAR and $200 \mathrm{ng}$ of CYLD into pCDNA3 kindly gifted by Gilles Courtois and supplemented by empty pCDNA3. Cells were lyzed $24 \mathrm{~h}$ after transfection and firefly luciferase activities measured using the Dual-Luciferase Reporter Assay System (Promega). Normalization for transfection efficiency in luciferase reporter assays was done by including a constant amount of a $\beta$-galactosidase expression plasmid in all transfections and measuring $\beta$-galactosidase activity. Each transfection was performed in triplicate, and three independent experiments were performed.

\section{RT-PCR experiments}

A guinea pig was obtained from Harlan. Treatment satisfied the requirements of the latest european directive 2010/63. Euthanasia was performed by a trained and authorized person ( $\left.{ }^{\circ} 69387512\right)$, administrating lethal dose of pentobarbital. Guinea pig total RNA was extracted from fresh or RNAlater (sigma) preserved tissues using Qiagen RNA easy kit. Macropus eugenii RNAs were a kind gift of Dr Kevin Nicholas (Melbourne University). Human RNA were obtained from Ambion: FirstChoice Human Total RNA Survey Panel for brain, mammary gland, kidney and skin. All RNAs were reverse transcribed using Superscript III Invitrogen. PCR fragments were amplified using an exon 1A specific primer or an exon $1 \mathrm{~B}$ specific primer and a reverse primer in exon 5, respectively: F-A TGGTCCCACGCAGCACTAGC, F-B 5'-GAGACCCGGGACGAACAGGC-3', R 5'-AGGGAGCGCTGTATCTTGAATGGG-3' for Cavia porcellus; F-A 5'-ATGGGACTCAGGACTACAAAGCA3' F-B 5'-ATGGGACTCAGGACTACAAAGC-3' R 5'AGGGTGCGCTGTATCTTGAATGGG-3' for Macropus eugenii, F-A 5' -ATGGGCCTCAGGACGACTAAACAGA TGG-3', F-B 5'-TGCGCGCAGATCATATGGTAAAGG AAC-3' and R 5' -ACCTGCGCAGAACCTTCTCCACG TC-3' for Homo sapiens. Sequences were deposited in Genebank with the following accession numbers: XXXX.

\section{RNAseq analysis}

The following datasets were downloaded from ENCODE website (http://genome.ucsc.edu/cgi-bin/hgTrackUi?hgsid= $346780313 \& \mathrm{c}=\mathrm{chr} 1 \& \mathrm{~g}=$ wgEncodeCaltechRnaSeq, human genome version hg19, GRCh37: GM92.paired.75 nt.; NHEK.paired.75 nt.200pb; GM78.paired.75 nt.200pb; H1ES.paired.75 nt.200pb; K562.paired.75 nt.200pb; MCF7.paired.75 nt.200pb; GM91.paired.75 nt.200pb. These cells lines were selected because of their relevance regarding the role of the Eda pathway in mammals: GM92, GM78 and GM91 cells are derived from mammary gland cell line, HE1S cells are derived from embryonic cell lines, NHEK are derived from epidermal keratinocytes, K562 cells are derived from leukemia and MCF7 cells are derived from mammary gland carcinoma.

To estimate the number of reads for each exon, we used the reads mapped on the human genome version hg 19 on ENCODE website. For each cell line and replicate, we extracted the BAM file corresponding table and discarded ambiguous reads. We then obtained specific reads for each exon $1 \mathrm{~A}$ and $1 \mathrm{~B}$ and for constitutive exon 6 as a control. As the number of read depends on exon size, we normalized the number of reads by exon size (228 bp for exon 1A, 245 bp for exon 1B and 426 bp for exon 6$)$ and checked that the number of reads was coherent by ensuring that exon $1 \mathrm{~A}+$ exon $1 \mathrm{~B}=$ exon 6 . True number of reads mapping to exon $A$ and exon $B$ were compared to expected numbers under the hypothesis that both isoforms were expressed at the same levels. This was done in each library, by using Chi-squared test comparing observed and expected distributions for the number 
of reads and corrected for multiple tests (7 tests). Results were unambiguous, as the 4 lines showing a significant difference in expression levels between A and B (NHEK $(p=10-15)$, H1ES (10-27), K562 (10-60), MCF7 (10-17)), also showed a clearly skewed fold change (more than 8-10 times in exon B compared to exon A).

\section{NF-кB reporter assays and Western Blotting}

Expression vectors for EDARADD B were kindly gifted by Asma Smahi and Céline Cluzeau and included 1) Full coding sequence of human EDARADD $B$ tagged with a C-terminal myc tag and cloned in pCDNA3.1 and 2) Full coding sequence of human EDARADD $B$ tagged with a $\mathrm{N}$-terminal HA tag and cloned in pCDNA 3. We amplified the EDARADD A N-terminal end by RT-PCR from human RNA and we used recombination to replace the sequence encoding the $\mathrm{B}$ peptide by the sequence encoding the A peptide directly in the above mentioned EDARADD B vectors (InFusion Cloning system, Clonetch).

For NF- $\mathrm{kB}$ activation assays, HEK cells grown in 24well plates were transfected using Exgen500 transfection reagent (Euromedex). Each well received $200 \mathrm{ng}$ of IgKLuc luciferase reporter (Clontech) kindly gifted by Asma Smahi and Céline Cluzeau as well as the above $E D A R A D D$ pcDNA3 vectors and empty pCDNA3 vector to give $1.0 \mu \mathrm{g}$ of DNA per well. Normalization for transfection efficiency in luciferase reporter assays was done by including a constant amount of a $\beta$-galactosidase expression plasmid in all transfections and measuring $\beta$-galactosidase activity. Cells were lyzed $24 \mathrm{~h}$ after transfection and luciferase activities measured using the Dual-Luciferase Reporter Assay System (Promega), according to the manufacturer's protocol. Each transfection was performed in triplicate, and three independent experiments were performed.

For Western Blotting, HEK cells grown in 6-well plates were transfected with $1.5 \mu \mathrm{g}$ of pCDNA3.1-EDARADD$m y c$ or empty pCDNA3 or $1.5 \mu \mathrm{g}$ pCDNA3.1-EDAR$A D D$-myc cotransfected with $750 \mathrm{ng}$ of EDAR or TRAF6 supplemented by empty pCDNA3 using Exgen500 transfection reagent (Euromedex). 24 hours or 48 hours after transfection, cells were harvested using ice-cold PBS and lysed in $50 \mu \mathrm{L}$ of RIPA buffer. Proteins were fractionated in $12 \% \mathrm{SDS} /$ polyacrylamide gel electrophoresis and transferred to a nitrocellulose membrane. Mouse anti-HA antibody (1:5000), mouse anti-myc antibody (1:5000) and rabbit anti-histone H3 (ab1791, Abcam) were used as primary antibodies and the reactive bands were detected via an ECL kit (Amersham Life Sciences).

\section{Fish husbandry}

Zebrafish and their embryos were handled according to standard protocols [40] and in accordance with the animal welfare committees of the Ecole Normale Supérieure de
Lyon. Zebrafish strains of $\mathrm{AB} / \mathrm{Tü}$ were reared and staged at $28.5{ }^{\circ} \mathrm{C}$ according to Kimmel and collaborators.

\section{RT-PCR for zebrafish experiments}

Total RNA was extracted from 25 embryos at various time points using the Nucleospin RNA II kit (MachereyNagel) according to the protocol for animal tissues and $1 \mu \mathrm{g}$ of RNA was reverse-transcribed using Moloney murine leukemia virus reverse transcriptase (Promega), followed by amplification of desired cDNA by PCR with Taq DNA polymerase with ThermoPol buffer (BioLabs). The ENSEMBL accession number for Danio rerio edaradd mRNA was ENSDART00000089916. Standard PCR primers for edaradd mRNA detection and splice blocking morpholino validation were as follows: For-edaradd, $5^{\prime}$ GCTCTGTGTTCCCACATCAA-3'; Rev-edaradd, 5' ACCAGGAGGAACAGAGCTGA-3'. The actin 1 gene was used as a control: For- $\beta$-actin1, $5^{\prime}$-AAGCAGGAG TACGATGAGTCTG-3'; and Rev- $\beta$-actin1, 5'-GGTA AACGCTCCTGGAATGAC-3' .

\section{Morpholino knockdown}

Morpholino oligonucleotides (edaradd MO-tsl (translation blocking): 5' -CACACATAGAAGAAGATCGTTGATC-3'; edaradd MO-spl (splice blocking): 5'-CACCAAGCGTATTTACTTACCAAAC-3') and the corresponding mismatch controls (MO sequence containing five base mismatches): edaradd MS-tsl: 5'-CAgAgATAcAAGAAcATCcTTGATC-3' and edaradd MS-spl: 5' -CACgAAcCGTATaTAgTTAgCAAAC-3') were supplied by Gene Tools and diluted in sterile water containing $0.05 \%$ phenol red. One-cell stage $A B / T \ddot{u}$ embryos were injected with $0.5 \mathrm{nl}$ of morpholino oligonucleotide dilution $(1 \mathrm{mM}$ for MO-tsl and $2 \mathrm{mM}$ for $\mathrm{MO}$-spl) and then incubated at $28.5{ }^{\circ} \mathrm{C}$ in $\mathrm{E} 3$ medium $(5 \mathrm{mM} \mathrm{NaCl}, 0.17 \mathrm{mM} \mathrm{KCl}$, $0.33 \mathrm{mM} \mathrm{CaCl} 2$, and $0.33 \mathrm{mM} \mathrm{MgSO} 4)$. The phenotypic observations of morphants were performed with a stereomicroscope or a light microscope (all from Leica) equipped with a digital camera. MO-injected embryos were routinely compared with corresponding MS-injected, phenol red-injected, and uninjected embryos.

\section{RNA preparation and rescue experiments}

Capped Homo sapiens EDARADD-A and $-B$ and Danio rerio edaradd were synthesized and purified from pCS2+:EDARADD- $A$ and $B$ and pCS2+:edaradd plasmids by using the mMESSAGE mMACHINE Sp6 kit (Ambion). For the rescue experiments, 25, 50 and $100 \mathrm{pg}$ of capped human EDARADD- $A,-B$ mRNA or capped zebrafish edaradd mRNA were co-injected with edaradd MO-tsl at the one-cell stage. These one-cell stage embryos were coinjected with MO-tsl and/or capped human EDARADD A or $\mathrm{B}$ mRNA with at least 70 embryos injected per 
condition. The barplot shows the percentage of each phenotype condition and is representative of at least 3 separate experiments with 3 different capped mRNA productions. Results are given as the mean \pm S.E. The significance of differences between mean values was evaluated by Student $\mathrm{t}$ test $\left(*, p<0.05 ;{ }^{* *}, p<0.01\right)$.

\section{Zebrafish staining and whole mount in situ hybridization} Alcian blue (cartilage) and Alizarin red (bone) double staining was performed as described [41] with minor modifications: staining was performed overnight on 5.5 dpf larvae with a solution containing $0.02 \%(\mathrm{w} / \mathrm{v})$ Alcian Blue 8GX, $100 \mathrm{mM} \mathrm{MgCl}_{2}$ and $0.005 \%$ (w/v) Alizarin red in $70 \% \mathrm{EtOH}$. Larvae were then bleached $20 \mathrm{~min}$ in $1.5 \%(\mathrm{v} / \mathrm{v}) \mathrm{H}_{2} \mathrm{O}_{2}$ and $1 \%(\mathrm{w} / \mathrm{v}) \mathrm{KOH}$ and then digested for $5 \mathrm{~min}$ in $1.67 \%(\mathrm{w} / \mathrm{v})$ trypsin.

Whole mount in situ hybridization was performed as previously described [42]. Staging was performing according to Kimmel. Sense and antisense RNA probes for each gene tested were prepared from partial cDNA. The development of endogenous pigments was inhibited by exposing embryos to 1-phenyl-2-thiourea (PTU) at a final concentration of $0.2 \mathrm{mM}$.

\section{Additional files}

Additional file 1: Figure $\mathbf{S 1}$. Genomic region of human EDARADD gene. Genomic region of the EDARADD gene in human (version hg19) vizualized in the UCSC genome browser (https://genome-euro.ucsc.edu/cgibin/hgTracks?db=hg19\&position=chr1\%3A236480456-236681365\&hgsid= 198526640_PuRKSKevW48nfe6o7ZmzCdqA0Kun). Genomic coordinates are the following: Chromosome 1: 236,348,262-236,484,914, exon 1A: Chr 1: 236,394,278-236,394,505, exon 1B: Chr 1: 236,395,416-236,395,660. In human ENCODE 7 cell, exon 1 A and B transcription initiation sites are embedded into a single H3K27ac peak, an epigenetic mark which is associated with initiation sites and active enhancers (green arrow). Human EST can be visualized above the gene organization, with $1 \mathrm{~A}$ or $1 \mathrm{~B}$ alternative first exon. Upstream, two human spliced EST match another H3K27ac peak (red arrow). They are composed of three exons matching this region and exons 2-6 of EDARADD. Such a transcript could possibly encode an EDARADD protein (that would then be initiated with an internal methionine as compared with $A$ and $B$ proteins, although it is not a consensus kozak sequence: gatcatATGG/gccA GccATGG). The functional significance of this putative EDARADD transcript is however very dubious. Not only ESTs matching these 3 additional exons are strongly underrepresented in EST databases as compared with EST matching exons $1 \mathrm{~A}$ et $1 \mathrm{~B}$, but they all come from testis, a tissue which is known for showing leaky transcription. We thus tend to believe that transcriptional activity at this site may rather reflect the presence of a distant enhancer of Edaradd (enhancers are known to be transcribed and show H3K27ac epigenetic mark as initiation sites) rather than the transcription of protein coding transcript. We nevertheless investigated the presence of a homologous transcript in other species. No upstream initiation site is found in mouse (EST database screening), suggesting that if this upstream H3K27ac peak corresponds to a third alternative promoter, this promoter is not conserved in mouse. Parts of the genomic region spanning the H3K27ac peak are conserved in the cow, but found on another chromosome than EDARADD (chr8 instead of 28); and no homologous region is found in dog; suggesting that if there is a third EDARADD alternative promoter, which we find unlikely, it would not be conserved in mammals. In conclusion, we did not consider this putative human third EDARADD transcript/promoter in the rest of the manuscript because its functional significance is dubious and moreover it does not seem conserved in mammals.
Additional file 2: Figure S2. Exon 1 outside mammals. Coding sequence of exon $1 \mathrm{~B}$ is well conserved in mammals and even in vertebrates whereas the one of exon $1 \mathrm{~A}$ is variable with a frequent loss of ATG (regarding A isoform, for an augmented dataset, see Fig. 1 and [Additional file 6 - Dataset S6]).

Additional file 3:Figure S3. EDARADD $A$ and $B$ do not exhibit any synergic or antagonistic effect for NF-KB activation. EDARADD A and $B$ were transfected and cotransfected in HEK cells as followed: EDARADD A $300 \mathrm{ng}$, EDARADD B $300 \mathrm{ng}$, EDARADD A $200 \mathrm{ng}$ with EDARADD B $100 \mathrm{ng}$, EDARADD A $100 \mathrm{ng}$ with EDARADD B $200 \mathrm{ng}$, EDARADD A $150 \mathrm{ng}$ with EDARADD B $150 \mathrm{ng}$. Each time, a NF-KB reporter plasmid that harbors NF-KB response elements upstream a luciferase reporter gene was cotransfected. The luciferase activity was normalized against the vector. The luciferase activity was assayed 24 hours after transfection.

Additional file 4: Figure S4. Details for experimental validation of edaradd knockdown with morpholinos and edaradd rescue experiments in zebrafish. A: edaradd mRNA level in zebrafish during embryonic development. edaradd mRNA level was analyzed by RT-PCR on whole embryos from 2.5 to $120 \mathrm{hpf}$. edaradd is highly detected as a maternal transcript before zygotic transcription starts. At $12 \mathrm{hpf}$, zygotic edaradd mRNA transcription starts and increases until $96 \mathrm{hpf}$. This result is representative of 3 independent experiments. B: Validation of Morpholino-induced edaradd downregulation. WT embryos were injected at one-cell stage with $4 \mathrm{ng}$ translation- (MO-tsl) or $8 \mathrm{ng}$ splice-blocking edaradd $\mathrm{MO}$ (MO-spl) and edaradd mRNA expression was analyzed by RT-PCR using specific primers to amplify DNA sequence either side of the targeted splice donor site (between exons 1 and 4) (see Fig. 5A for primer and morpholino localization). At $48 \mathrm{hpf}$, we observed an absence of PCR product in MO-spl-injected embryos when compared to MS-spl-, MO-tsl- and MS-tsl-injected embryos demonstrating that the MO-spl efficiently blocked edaradd splicing. As expected, neither injection with translation blocking $\mathrm{MO}$ (MO-tsl) nor with its mutated version (MS-tsl) impacted edaradd mRNA levels. This result is representative of 3 independent experiments. C: Knockdown of edaradd expression with MO-tsl or MO-spl. Percentages for the various phenotypic groups obtained following MO-tsl injection were determined and are representative of 3 independent experiments; each experiment was performed with at least 70 injected embryos. D: Determination of human EDARADD A and B role through rescuing experiments in zebrafish. One-cell stage embryos were co-injected with MO-tsl and/or capped human EDARADD A or B or zebrafish edaradd mRNA. At least 70 embryos were injected per condition and the results are expressed as the percentage of each phenotypic groups observed per condition. Results are representative of 6 separate experiments performed with 2 different batches of mRNA. Statistical analyses have been performed on the severe phenotypic condition. E: Overexpression of human EDARADD A or B isoforms or zebrafish Edaradd has no obvious effect on skeletal development. WT embryos were injected with $100 \mathrm{pg}$ of capped human EDARADD A or B mRNA or zebrafish edaradd mRNA at one-cell stage and skeletal morphogenesis was analyzed through alcian blue and alizarin red staining at $5.5 \mathrm{dpf}$. A representative ventral view of the head of a larvae is shown for each condition and a zoom on the teeth is shown on the right panel (scale bar: $100 \mu \mathrm{m})$.

Additional file 5: Figure S5. Expression of Eda and Edar in the pharyngeal region during zebrafish embryogenesis determined using whole mount in situ hybridization. A: Coexpression of eda and edar at $60 \mathrm{hpf}$ in the pharyngeal region in lateral view (up) and ventral view (low). B: Expression pattern of eda from 48 to $80 \mathrm{hpf}$. For each stage a lateral view is shown with, as an inset, a ventral view. No expression was detected before 48hpf. C: Expression pattern of edar from 24 to $80 \mathrm{hpf}$. For each stage a lateral view is shown with, as an inset, a ventral view. No expression was detected before $24 \mathrm{hpf}$.

Additional file 6: Dataset S6. Sequences dataset of the exon 1A region. Alignment of the exon $1 \mathrm{~A}$ region obtained by BLAST or sequencing (see material and method for details) in Seaview software. Human TSS (Transcription Start Site) and the two ATG are annotated. 


\section{Authors' contributions}

AS conceived and performed the experiments and drafted the manuscript. $\mathrm{EL}$ and FR conceived and performed the zebrafish morpholino experiments. PC obtained part of the exon 1A dataset. MS conceived the RNAseq data analysis. MT performed in situ hybridization experiments. DD and TO designed in vitro translation experiments. SP and VL conceived the experiments, revised the manuscript and conducted the whole work. All authors read and approved the final manuscript.

\section{Acknowledgments}

This work was supported by the Center National pour la Recherche Scientifique, the Ecole Normale Supérieure de Lyon, the Région Rhône-Alpes, the Fondation ARC for Reasearch on Cancer. We thank Denis Headon (The Roslin Institute, University of Edinburgh) for kindly giving us the NF-kB reporter plasmid, Céline Cluzeau and Asma Smahi (INSERM U781 Hôpital Necker-Enfants Malades) for giving us the HA-EDARADD $B$ and EDARADD B -myc plamids and Gilles Courtois (INSERM U1038/Gen\&Chem, CEA Grenoble) for giving us the CYLD plasmid. For mammalian tissues, we thank François Catzeflis and the Institut des Sciences de l'Evolution of Montpellier. Macropus RNA was kindly provided by Dr Kevin Nicholas from Melbourne University. We thanks the PRECI and Laure Bernard for the zebrafish facility. We thank anonymous reviewers for their helpful criticisms on our manuscript and Anamaria Necsuela for the RNAseq mammal dataset. We thank Joanne Burden for helpful reading of the manuscript.

\section{Author details}

${ }^{1}$ Institut de Génomique Fonctionnelle de Lyon, UMR 5242 du CNRS, Université de Lyon, Université Claude Bernard Lyon 1, Ecole Normale Supérieure de Lyon, 46 Allée d'Italie, 69364 Lyon Cedex 07, France. ${ }^{2}$ Laboratoire de Biométrie et Biologie Évolutive, CNRS UMR5558, Université de Lyon, Universite Claude Bernard Lyon 1, Villeurbanne, France. ${ }^{3} \mathrm{CIRI}$, International Center for Infectiology Research, Université de Lyon, INSERM U1111, Ecole Normale Supérieure de Lyon, Lyon, France.

Received: 27 December 2014 Accepted: 29 May 2015 Published online: 02 July 2015

\section{References}

1. Canestro C, Yokoi H, Postlethwait JH. Evolutionary developmental biology and genomics. Nat Rev Genet. 2007:8(12):932-42.

2. Pires-daSilva A, Sommer RJ. The evolution of signalling pathways in animal development. Nat Rev Genet. 2003;4(1):39-49.

3. Muller GB. Evo-devo: extending the evolutionary synthesis. Nat Rev Genet. 2007;8(12):943-9.

4. Barbosa-Morais NL, Irimia M, Pan Q, Xiong HY, Gueroussov S, Lee LJ, et al. The evolutionary landscape of alternative splicing in vertebrate species. Science. 2012;338(6114):1587-93.

5. Merkin J, Russell C, Chen P, Burge CB. Evolutionary dynamics of gene and isoform regulation in Mammalian tissues. Science. 2012;338(6114):1593-9.

6. Licatalosi DD, Darnell RB. RNA processing and its regulation: global insights into biological networks. Nat Rev Genet. 2010;11(1):75-87.

7. Kalsotra A, Cooper TA. Functional consequences of developmentally regulated alternative splicing. Nat Rev Genet. 2011;12(10):715-29.

8. Wang ET, Sandberg R, Luo S, Khrebtukova I, Zhang L, Mayr C, et al. Alternative isoform regulation in human tissue transcriptomes. Nature. 2008;456(7221):470-6.

9. Kelemen $\mathrm{O}$, Convertini $\mathrm{P}$, Zhang $Z$, Wen $Y$, Shen $M$, Falaleeva $\mathrm{M}$, et al. Function of alternative splicing. Gene. 2013;514(1):1-30.

10. Kim E, Magen A, Ast G. Different levels of alternative splicing among eukaryotes. Nucleic Acids Res. 2007;35(1):125-31.

11. Nilsen TW, Graveley BR. Expansion of the eukaryotic proteome by alternative splicing. Nature. 2010;463(7280):457-63.

12. Chen L, Bush SJ, Tovar-Corona JM, Castillo-Morales A, Urrutia AO. Correcting for Differential Transcript Coverage Reveals a Strong Relationship between Alternative Splicing and Organism Complexity. Mol Biology Evol. 2014;31(6):1402-13.

13. Sadier A, Viriot L, Pantalacci S, Laudet V. The ectodysplasin pathway: from diseases to adaptations. Trends Genet. 2014;30(1):24-31.

14. Lefebvre S, Mikkola ML. Ectodysplasin research-where to next? Semin Immunol. 2014;26(3):220-8.
15. Fujimoto A, Kimura R, Ohashi J, Omi K, Yuliwulandari R, Batubara L, et al. A scan for genetic determinants of human hair morphology: EDAR is associated with Asian hair thickness. Hum Mol Genet. 2008;17(6):835-43.

16. Colosimo PF, Hosemann KE, Balabhadra S, Villarreal Jr G, Dickson M, Grimwood J, et al. Widespread parallel evolution in sticklebacks by repeated fixation of Ectodysplasin alleles. Science. 2005:307(5717):1928-33.

17. Morlon A, Munnich A, Smahi A. TAB2, TRAF6 and TAK1 are involved in NF-kappaB activation induced by the TNF-receptor, Edar and its adaptator Edaradd. Hum Mol Genet. 2005;14(23):3751-7.

18. Pantalacci S, Chaumot A, Benoit G, Sadier A, Delsuc F, Douzery EJ, et al. Conserved features and evolutionary shifts of the EDA signaling pathway involved in vertebrate skin appendage development. Mol Biol Evol. 2008;25(5):912-28

19. Headon DJ, Emmal SA, Ferguson BM, Tucker AS, Justice MJ, Sharpe PT, et al. Gene defect in ectodermal dysplasia implicates a death domain adapter in development. Nature. 2001;414(6866):913-6.

20. Koppinen P, Pispa J, Laurikkala J, Thesleff I, Mikkola ML. Signaling and subcellular localization of the TNF receptor Edar. Exp Cell Res. 2001;269(2):180-92.

21. Harris MP, Rohner N, Schwarz H, Perathoner S, Konstantinidis P, Nusslein-Volhard C. Zebrafish eda and edar mutants reveal conserved and ancestral roles of ectodysplasin signaling in vertebrates. PLoS Genet. 2008;4(10):e1000206.

22. Sadier A, Viriot L, Pantalacci S, Laudet V. The ectodysplasin pathway: from diseases to adaptations. Trends Genet. 2014;30(1):24-31.

23. Cluzeau C, Hadj-Rabia S, Jambou M, Mansour S, Guigue P, Masmoudi S, et al. Only four genes (EDA1, EDAR, EDARADD, and WNT10A) account for $90 \%$ of hypohidrotic/anhidrotic ectodermal dysplasia cases. Hum Mutat. 2011;32(1):70-2

24. Bal E, Baala L, Cluzeau C, El Kerch F, Ouldim K, Hadj-Rabia S, et al. Autosomal dominant anhidrotic ectodermal dysplasias at the EDARADD locus. Hum Mutat. 2007:28(7):703-9.

25. Bergendal B, Klar J, Stecksen-Blicks C, Norderyd J, Dahl N. Isolated oligodontia associated with mutations in EDARADD, AXIN2, MSX1, and PAX9 genes. Am J Med Genet A. 2011;155A(7):1616-22

26. Koguchi-Yoshioka H, Wataya-Kaneda M, Yutani M, Nakano H, Sawamura D, Katayama I. Partial anhidrosis demonstrated by Q-SART in a patient with a novel mutation in the EDARADD gene. J Eur Acad Dermatol Venereol. 2014:29(6);jdv.12493.

27. Wolpert C, Schimpf R, Veltmann C, Borggrefe M. Short QT syndrome. Herz. 2007:32(3):206-10

28. Aigler SR, Jandzik D, Hatta K, Uesugi K, Stock DW. Selection and constraint underlie irreversibility of tooth loss in cypriniform fishes. Proc Natl Acad Sci U S A. 2014;111(21):7707-12.

29. Bornert F, Choquet $\mathrm{P}$, Gros Cl, Aubertin G, Perrin-Schmitt F, Clauss F, et al. Subtle Morphological Changes in the Mandible of Tabby Mice Revealed by Micro-CT Imaging and Elliptical Fourier Quantification. Front Physiol. 2011;2:15.

30. Heath J, Langton AK, Hammond NL, Overbeek PA, Dixon MJ, Headon DJ. Hair follicles are required for optimal growth during lateral skin expansion. J Invest Dermatol. 2009:129(10):2358-64.

31. Gomez C, Chua W, Miremadi A, Quist S, Headon DJ, Watt FM. The interfollicular epidermis of adult mouse tail comprises two distinct cell lineages that are differentially regulated by Wnt, Edaradd, and Lrig1. Stem Cell Rep. 2013;1(1):19-27.

32. Mikkola ML. TNF superfamily in skin appendage development. Cytokine Growth Factor Rev. 2008;19(3-4):219-30

33. Mou C, Jackson B, Schneider P, Overbeek PA, Headon DJ. Generation of the primary hair follicle pattern. Proc Natl Acad Sci U S A. 2006;103(24):9075-80.

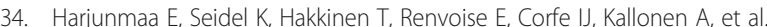
Replaying evolutionary transitions from the dental fossil record. Nature. 2014:512(7512):44-8

35. Kangas AT, Evans AR, Thesleff I, Jernvall J. Nonindependence of mammalian dental characters. Nature. 2004;432(7014):211-4.

36. Gomez-Rodriguez et al. Evolutionary and developmental dynamics of the dentition in muroidea and dipodoide.pdf;2011;13(4), 361-36.

37. Meredith RW, Janecka JE, Gatesy J, Ryder OA, Fisher CA, Teeling EC, et al. Impacts of the Cretaceous Terrestrial Revolution and KPg extinction on mammal diversification. Science. 2011;334(6055):521-4.

38. Edgar RC. MUSCLE: multiple sequence alignment with high accuracy and high throughput. Nucleic Acids Res. 2004;32(5):1792-7.

39. Soto Rifo R, Ricci EP, Decimo D, Moncorge O, Ohlmann T. Back to basics: the untreated rabbit reticulocyte lysate as a competitive system to 
recapitulate cap/poly(A) synergy and the selective advantage of IRES-driven translation. Nucleic Acids Res. 2007;35(18):e121.

40. Kimmel CB, Ballard WW, Kimmel SR, Ullmann B, Schilling TF. Stages of embryonic development of the zebrafish. Dev Dyn. 1995;203(3):253-310.

41. Walker MB, Kimmel CB. A two-color acid-free cartilage and bone stain for zebrafish larvae. Biotech Histochem. 2007:82(1):23-8.

42. Thisse B, Heyer V, Lux A, Alunni V, Degrave A, Seiliez I, et al. Spatial and temporal expression of the zebrafish genome by large-scale in situ hybridization screening. Methods Cell Biol. 2004;77:505-19.

43. Hall BK. Homology. The hierarchical basis of comparative biology. San Diego: Academic Press; 1994.

44. Gerstein MB, Lu ZJ, Van Nostrand EL, Cheng C, Arshinoff BI, Liu T, et al. Integrative analysis of the Caenorhabditis elegans genome by the modENCODE project. Science. 2010;330(6012):1775-87.

\section{Submit your next manuscript to BioMed Central and take full advantage of:}

- Convenient online submission

- Thorough peer review

- No space constraints or color figure charges

- Immediate publication on acceptance

- Inclusion in PubMed, CAS, Scopus and Google Scholar

- Research which is freely available for redistribution 